Gazi University
Journal of Science
http://dergipark.gov.tr/gujs

\title{
Urban Road Network Maintenance Scheduling Using Ant Colony Optimization
}

\author{
Ilyas Cihan AKSOY ${ }^{1, *}$, Mehmet Metin MUTLU ${ }^{2}$, Yalcin ALVER ${ }^{3}$ \\ ${ }^{I}$ Department of Civil Engineering, Faculty of Engineering, Karamanoglu Mehmetbey University, Karaman, Turkey \\ ${ }^{2}$ Department of Civil Engineering, Faculty of Engineering, Aydin Adnan Menderes University, Aydin, Turkey \\ ${ }^{3}$ Department of Civil Engineering, Faculty of Engineering, Ege University, Izmir, Turkey
}

\author{
Highlights \\ - The study presents a road maintenance scheduling model based on ACO. \\ - The model aims to obtain a schedule minimizing total travel time during the maintenance. \\ - We embed minor modifications into ACO to adapt into the maintenance scheduling problem. \\ - Tests are carried out on a small-scale fictitious network. \\ - The model provides a significant reduction in the total travel time.
}

\section{Article Info}

Received:02 Sep 2020

Accepted:20 Dec 2020

\section{Keywords}

Maintenance scheduling

Bi-level optimization

Ant colony optimization

\begin{abstract}
In this study, an optimization model for road maintenance scheduling is proposed. The proposed model determines a maintenance schedule for a given set of links in an urban road network, that minimizes the total travel time during the maintenance period under a set of assumptions and a particular crew number constraint. It is assumed that all lanes of a road to be repaired are closed to traffic during the maintenance. Ant Colony Optimization (ACO), a suitable algorithm for discrete transportation problems, is employed in the proposed model. The model generates a set of roads to be closed for each maintenance day. The total travel time during the whole maintenance period is calculated using the deterministic user equilibrium assignment model. The proposed model is applied to a test network, and more efficient schedules in terms of total travel time are obtained compared to randomly generated schedules
\end{abstract}

\section{INTRODUCTION}

Maintenance of urban roads involves closure of a road partly or entirely, which leads to traffic congestion due to a reduced capacity of the network and increases travel times of network users. To mitigate these negative impacts, a well-planned maintenance schedule is necessary to use network capacity as efficiently as possible during the maintenance period.

Maintenance of a road link forces the users of that road link to change their regular routes to minimize their travel time, which results in increased flows in other roads in the network. Therefore, in addition to the users of road links to be maintained, all users of the network are likely to encounter higher congestion leading to higher travel times. In a case of multiple roads to be repaired, considering crew number constraints, it is vital to determine which roads should be repaired simultaneously.

In road network maintenance scheduling problems (RNMSP), all candidate schedules result in different network topologies due to link closures. Since users make different travel choices for each unique network structure, different schedules cause different link flows and travel times. Thus, to consider network users' reactions to different network structures, maintenance scheduling of roads should be formulated as a bilevel model. In this study, the upper level of the bi-level structure aims to obtain a better schedule iteratively using an optimization method. The lower-level model is a traffic assignment model that calculates the link flows and the total travel cost due to schedule determined in the upper-level model. 
RNMSPs belong to the class of time-consuming NP-hard problems, with combinatorial decision variables. The feasible solution space of such a problem expands exponentially depending on crew number and link number to be repaired. Consequently, RNMSPs are challenging to solve using traditional methods. Therefore, metaheuristics, well-known optimization tools for solving such problems, are convenient solution techniques.

There are several studies concerning road network maintenance scheduling in the literature up to date. Firstly, [1] presents a model minimizing the total traffic delay with the help of the Genetic Algorithm (GA) by allocating maintenance teams to a set of repair requests of lane sections, subject to a constraint of standby hours of maintenance teams. Traffic delay calculation is based on the work of [2]. The model is tested on 20 road sections, identified by surveys in Singapore, to be repaired by three maintenance teams. Three runs of GA are completed with 1000 iterations, and the best solution gives a delay value of 1.148 vehicle-hours.

[3] employs a Tabu Search algorithm to determine the combinations of the roads to be repaired to decrease total traffic delay. Fitness values of candidate solutions are calculated using a static traffic assignment model. The method is implemented on six links of Columbus network, and the best solution decreases the total traffic delay by $0.27 \%$ when compared to the case of no congestion.

[4] aims to develop a maintenance schedule model, minimizing the total travel time in the case of lane closures under a constraint of standby hours of maintenance teams, using a hybrid model of GA and PARAMICS software to estimate the total travel time. The hybrid model is applied to a network consisting of 74 nodes, 160 links, and 13 zones. GA, with a population size of 4 and 20 generations, is executed nine times to distribute three maintenance teams to 10 repair requests. The best solution obtains a reduction of $5.1 \%$ in the total travel time when compared to the initial values.

[5] presents a maintenance scheduling model that gives priority to the most critical link among damaged links using Greedy Search Algorithm. The model is applied to eight links of the Sioux Falls network. The results are compared with the exact solutions over the selected four links to prove the correctness of the Greedy Algorithm.

[6] handles road maintenance scheduling problem using dynamic programming model to minimize the network users' delay caused by the maintenance subject to the budget constraint. The model is performed on a small-sized Nguyen-Dupuis network, a large-sized Sioux-Falls network.

[7] proposes a day-based work zone scheduling model in the case of works to be done in more than one day considering the change in traffic, utilizing day-to-day traffic assignment. The proposed model is implemented on a network composed of 16 nodes and 24 links, with six links to be repaired. The model aims to minimize the increment of travel costs caused by the maintenance works. The model employs GA for optimization.

[8] address the problem of minimizing total system travel time employing a new branch-and-price algorithm. They calculate the flows and travel times of the links according to the deterministic user equilibrium conditions. The proposed model is applied to Sioux Falls, BerlinMPR, and Barcelona networks.

This study proposes a maintenance scheduling model minimizing the total travel time during the maintenance period. Road links to be repaired are assigned to days under the constraint of crew number. To obtain an optimal schedule, Ant Colony Optimization (ACO), a suitable metaheuristic for discrete transportation problems, is employed. Several minor modifications are embedded into ACO to adapt our problem. The tests are carried out on a small-scale hypothetical network. This paper is organized as follows: the problem description and solution method are described in Section 2. Section 3 gives details of the results. Lastly, Section 4 presents a general review and proposes future studies. 


\section{METHODOLOGY}

We developed a road network maintenance scheduling model for a given set of links in a network to obtain an optimal maintenance schedule, which minimizes the total travel time subject to a constraint of maintenance crew number by adopting a bi-level model approach. The assumptions underlying the proposed model are as follows:

1. Each maintenance crew can repair only one road link in a day.

2. Maintenance crews are available every day during the maintenance period.

3. All lanes of a road are closed during the maintenance day.

4. The maintenance duration of a link is less than one day.

5. The number of maintenance crews is limited.

Let us denote the number of roads to be repaired and the number of maintenance crews as $n$ Link and $n C r e w$, respectively. It is evident that the required number of days, $n D a y$, for the whole maintenance period is equal to $n$ Link/nCrew under the mentioned assumptions.

The bi-level model proposed in this study employs ACO developed by Dorigo \& Caro (1999) to overcome such a maintenance scheduling problem [9]. ACO is based on the behavior of the ant colony with the size of $n A n t$. While searching for a path between food source and nest, ants deposit pheromone, a chemical component, on paths. The amount of pheromone of less-cost paths is likely to be more. The following ants select their paths according to probability, depending on the updated amount of pheromone, tau, and the power of pheromone, $\gamma$. To hinder the amount of pheromone to rise limitlessly, the pheromone trail decay coefficient, $\rho$, is applied to tau. In this study, ACO is used as a tool distributing a set of variables to different subsets instead of selecting a set from a vast search space. To that end, a minor modification is applied to ACO by authors.

A measurable objective is required to evaluate the fitness of a schedule determined at the upper level. The model proposed in this study determines a maintenance schedule minimizing the total travel time for all users using the network during the maintenance period without considering the mobilization cost of maintenance crews and tools. Total travel time for all network users is based on the sum of travel times during the maintenance period, as described in the following equation:

$$
T T T=\sum_{d \in D} \sum_{a \in A} t_{d, a} \times x_{d, a}
$$

$T T T$ : total travel time for all users in the network

$D$ : set of maintenance days

$A$ : set of road links in the network

$d$ : index of the maintenance day

$a$ : index of the link

$t_{d, a}:$ the travel time of road link $a$ in the maintenance day $d$

$x_{d, a}$ : the flow of road on link $a$ in the maintenance day $d$.

In the lower-level, the traffic assignment process is applied to the network for each maintenance day to calculate total travel time, since a different set of roads is closed for each different day. The traffic assignment model of this study adopts Deterministic User Equilibrium (DUE) conditions and is solved using the Frank-Wolfe Algorithm. Congested travel time of each road in the network is based on the BPR function as in the following equation;

$$
t=t_{0} \times\left(1+\alpha \times\left(\frac{V}{C}\right)^{\beta}\right)
$$


$t$ : Congested travel time of link

$t_{0}$ : Free flow travel time of link

$V:$ Traffic volume on link

$C:$ Effective capacity of the link

$\alpha, \beta$ : Calibration parameters.

The steps of the road network maintenance scheduling model based on the modified ACO are given as follows;

Step 0: Initialization. Determine input data

Step 0.1: Input. $z, n A n t$, tau $_{0}, \rho, \gamma, Q, n C r e w, n D a y, n L i n k$.

Step 0.2: Prepare Links. Give id to the links to be repaired from 1 to $n L i n k s$, compose $\mathbf{R}=\{1,2$, ...,nLinks\}.

Step 0.3: Pheromone Matrix. Create tau $\mathrm{m}_{\mathrm{m}, \mathrm{n}}=\operatorname{tau}_{0}, \forall m, n \in\{1,2, \ldots, n$ Links $\}$

Step 1: Create Ant Colony. Generate an ant colony with size $n A n t$, each ant $\mathbf{A n t}_{i}=\left\{A n t_{i, 1,1}, A n t_{i, 1,2}, \ldots\right.$, $A n t_{i, 1, k}$, Ant $_{i, 2,1}$, Ant $_{i, 2,2, \ldots,}$ Ant $\left._{i, j, k}\right\}, \forall j \in\{1,2, \ldots, n D a y\}, \forall k \in\{1,2, \ldots, n C r e w\}$.

Step 2: Loop. For each $\mathbf{A n t}_{i}$ in the colony.

Step 2.1: Loop. For each Ant $\mathbf{A}_{i, j}=\left\{A n t_{i, j, 1}, A n t_{i, j, 2}, \ldots, A n t_{i, j, n C r e w}\right\}$, of $\mathbf{A n t}_{i}$ in the maintenance day $j$.

Step 2.1.1: Loop. For each $A n t_{i, j, k}$ of $\mathbf{A n t}_{i, j}$

Step 2.1.1.1: Selection. If $k=1, A n t_{i, j, 1}=\min \left\{\mathbf{R}\right.$ - $\left.\mathbf{A n t}_{i}\right\}$. Otherwise, go to Step 2.1 .2

Step 2.1.1.2: Selection. Select a road link to be repaired for $A n t_{i, j, k}$ in maintenance day $j$

for crew $k$ from $\left\{\mathbf{R}-\mathbf{A n t}_{i}\right\}$ by tau and $\gamma$.

Step 2.1.2: Sorting. Sort $\mathbf{A n t}_{i, j}$ according to id number of links in $\mathbf{R}$.

Step 2.2: Traffic Assignment. Assign users to network for each maintenance day $j$, considering link closures.

Step 2.3: Cost Calculation. Calculate the total travel cost, $c_{i}$, of Ant ${ }_{i}$ by Eq. (1).

Step 2.4: Replacement. If $c_{i}<c_{\text {best }}$ that the algorithm obtains the best total travel cost until that moment, update the best schedule by Ant . $_{\text {. }}$

Step 3: Update Pheromone. Update tau for each $A n t_{i, j, k}$ of each $\mathbf{A n t}_{i}$ by the ratio of $Q$ to $c_{i}$.

Step 4: Evaporation. Update tau by $\rho$.

Step 5: Reset. Ant $_{i}=\emptyset$ for all ants.

Step 6: Termination. If maximum iteration number $z$ is reached, Stop. Otherwise, go to Step 2.

\section{COMPUTATIONAL EXPERIMENTS AND RESULTS}

A hypothetical grid network consisting of 16 nodes and 48 links, is used to evaluate the model. The test network and the demand list are given in Figure 1. Link no, free-flow travel time in minutes, and effective capacity of each link of the test network are depicted on the corresponding links in order.

It is assumed that the links 4, 7, 8, 10, 13, 18, 24, 29, 32, 33, 38, and 46, which are indicated by dashed lines in Figure 1, are required to be repaired by three maintenance crews. The number of days that maintenance is required to be done is equal to four under the model assumptions that are previously mentioned. The number of all feasible schedules is 15,400 , according to Equation (3)

$$
s=\frac{C_{n \text { Crew }}^{n \text { Link }} \times C_{n \text { Crew }}^{n \text { nink } n \text { Crew }} \times C_{n \text { Crew }}^{n \text { Link } 2 \times n \text { Crew }} \times \ldots \times C_{n \text { Crew }}^{n \text { Crew }}}{\left(\frac{n \text { Link }}{n C r e w}\right) !} .
$$

ACO and traffic assignment algorithms are coded in MATLAB. All parameters of ACO, traffic assignment, and BPR function are set as; $\beta=4, \alpha=0.15, n A n t=50, z=50, \tan _{0}=5, \gamma=1, \rho=0.1, Q=100,000, n$ Crew $=3, n$ Day $=4, n$ Link $=12$. 


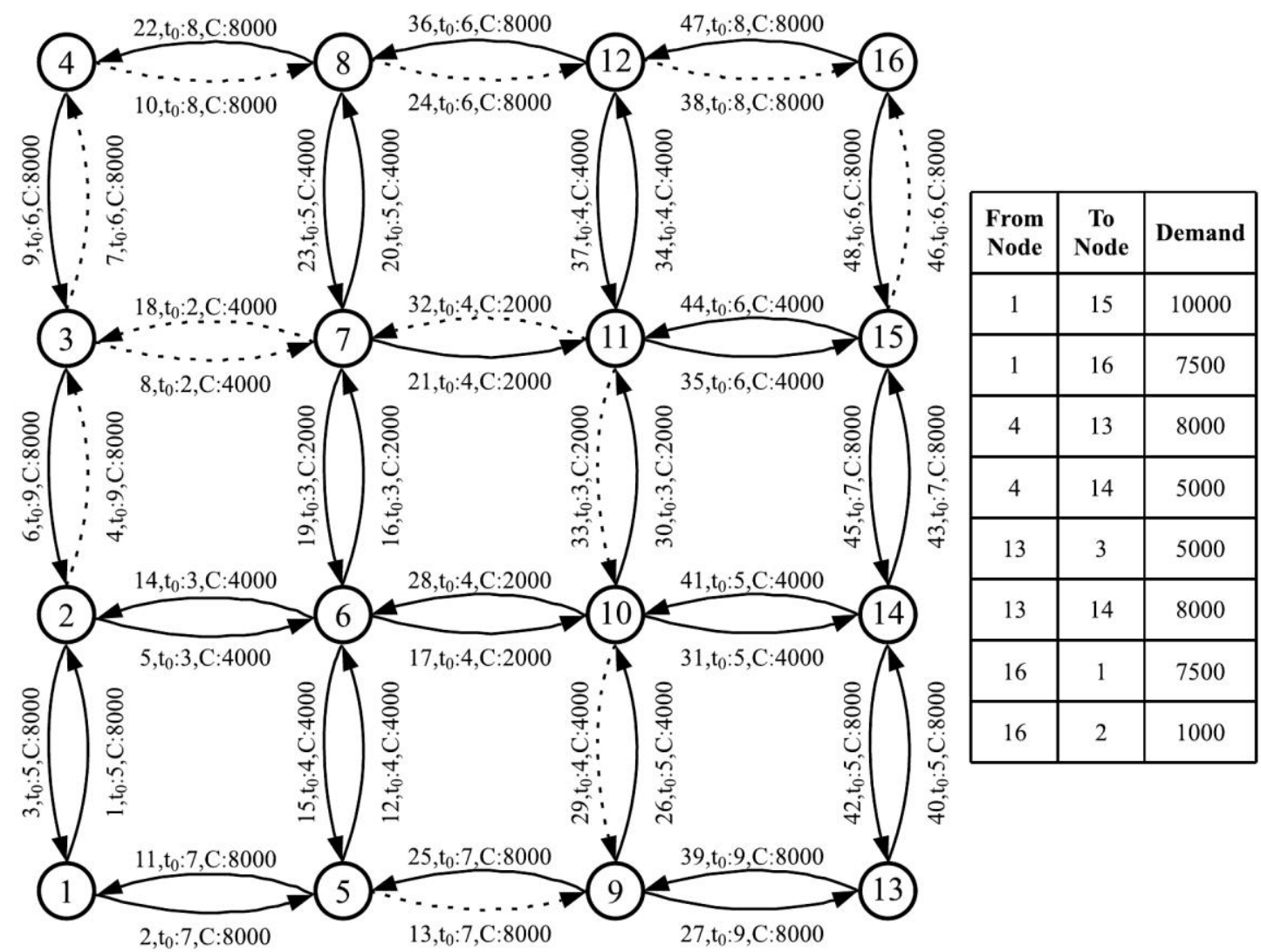

Figure 1. $4 \times 4$ Grid Test Network

For the given network with no road closures, under the user equilibrium conditions, the total travel time is $3,291,765$ minutes for one day and 13,167,062 minutes for the whole maintenance period of four days. Table 1 presents ten randomly chosen schedules, corresponding total travel times of four days, and increases in the total travel time compared to no-road-closure case. The average total travel time of ten schedules is $51,627,351$ minutes.

Table 1. The assignment results of ten randomly chosen solution sets

\begin{tabular}{|l|l|l|l|l|l|l|}
\hline \multirow{2}{*}{ Set No } & \multirow{2}{*}{ Total Travel Time (min) } & \multirow{2}{*}{ Increase (\%) } & Solution Set \\
\cline { 4 - 7 } & & & Day 1 & Day 2 & Day 3 & Day 4 \\
\hline 1 & $80,534,332$ & 511.63 & $\{4,7,29$ & $8,32,46$ & $10,13,24$ & $18,33,38\}$ \\
\hline 2 & $24,431,329$ & 85.55 & $\{4,8,38$ & $7,18,32$ & $10,13,29$ & $24,33,46\}$ \\
\hline 3 & $18,322,400$ & 39.15 & $\{4,10,29$ & $7,24,33$ & $8,18,32$ & $13,38,46\}$ \\
\hline 4 & $79,486,099$ & 503.67 & $\{4,10,33$ & $7,8,29$ & $13,24,32$ & $18,38,46\}$ \\
\hline 5 & $81,130,618$ & 516.16 & $\{4,7,18$ & $8,29,32$ & $10,33,38$ & $13,24,46\}$ \\
\hline 6 & $79,683,189$ & 505.17 & $\{4,13,24$ & $7,8,33$ & $10,29,38$ & $18,32,46\}$ \\
\hline 7 & $81,643,513$ & 520.06 & $\{4,32,46$ & $7,10,29$ & $8,18,33$ & $13,24,46\}$ \\
\hline 8 & $24,837,039$ & 88.63 & $\{4,7,10$ & $8,29,33$ & $13,18,32$ & $24,38,46\}$ \\
\hline 9 & $22,749,964$ & 72.78 & $\{4,13,29$ & $7,8,46$ & $10,24,32$ & $18,33,38\}$ \\
\hline 10 & $23,455,034$ & 78.13 & $\{4,13,33$ & $7,8,24$ & $10,18,38$ & $29,32,46\}$ \\
\hline
\end{tabular}

The proposed model is executed 30 times on the hypothetical test network. Table 2 shows 12 unique solution sets obtained, corresponding total travel times, frequencies of being obtained, the percent increase in the total travel time according to the no-road-closure case for each unique solution. The best schedule concerning total travel time achieved by the model results in a total travel time of $16,810,173$ minutes, which is achieved five times among 30 replications. The average total travel time of 30 solutions is $16,922,607$ minutes, while the worst solution has a total travel time of 17,041,193 minutes. 
Table 2. The results of 30 optimization runs

\begin{tabular}{|c|c|c|c|c|c|c|}
\hline \multicolumn{4}{|c|}{ Solution Set } & \multirow{2}{*}{ Total Travel Time(min) } & \multirow{2}{*}{ Increase $(\%)$} & \multirow{2}{*}{ Frequency } \\
\hline Day 1 & Day 2 & Day 3 & Day 4 & & & \\
\hline$\{4,7,10$ & $8,29,33$ & $13,18,32$ & $24,38,46\}$ & $16,810,173$ & 27.67 & 5 \\
\hline$\{4,38,46$ & $7,13,18$ & $8,29,33$ & $10,24,32\}$ & $16,899,431$ & 28.35 & 4 \\
\hline$\{4,38,46$ & $7,13,29$ & $8,18,33$ & $10,24,32\}$ & $16,977,540$ & 28.94 & 2 \\
\hline$\{4,7,10$ & $8,32,33$ & $13,18,29$ & $24,38,46\}$ & $17,022,659$ & 29.28 & 3 \\
\hline$\{4,38,46$ & $7,13,18$ & $8,29,32$ & $10,24,33\}$ & $16,971,372$ & 28.89 & 2 \\
\hline$\{4,7,10$ & $8,18,32$ & $13,29,33$ & $24,38,46\}$ & $17,017,230$ & 29.24 & 1 \\
\hline$\{4,38,46$ & $7,10,24$ & $8,29,33$ & $13,18,32\}$ & $16,828,707$ & 27.81 & 2 \\
\hline$\{4,7,8$ & $10,29,33$ & $13,18,32$ & $24,38,46\}$ & $16,894,843$ & 28.31 & 4 \\
\hline$\{4,38,46$ & $7,13,29$ & $8,18,32$ & $10,24,33\}$ & $16,897,524$ & 28.33 & 2 \\
\hline$\{4,10,29$ & $7,13,18$ & $8,32,33$ & $24,38,46\}$ & $16,953,066$ & 28.75 & 2 \\
\hline$\{4,10,33$ & $7,13,29$ & $8,18,32$ & $24,38,46\}$ & $17,033,720$ & 29.37 & 2 \\
\hline$\{4,38,46$ & $7,10,24$ & $8,32,33$ & $13,18,29\}$ & $17,041,193$ & 29.42 & 1 \\
\hline
\end{tabular}

The results show that the proposed scheduling model can obtain schedules leading to a significant reduction in total travel time when compared to randomly generated schedules. The best of the randomly generated schedules has a total travel time of 18,322,400 minutes, while the best one of the optimal solutions has a total travel time of 16,810,173 minutes during the maintenance period. Evaluation of the results shows that the proposed scheduling optimization model can be considered to be successful.

\section{CONCLUSION}

This study proposes a bi-level maintenance scheduling model minimizing the total travel time for private car users in a road network during the maintenance period, subject to a crew number constraint. The model utilizes ACO in the upper-level model to solve such a complex and time-consuming problem. The cost function of the minimization problem, the total travel time of road network users, is calculated performing user equilibrium traffic assignment in each iteration with a modified network topology based on the upperlevel model decisions.

The proposed model is performed on a hypothetical network with 16 nodes and 48 links. The number of links that are required to be repaired by three maintenance crews is 12 . The best of solutions generated by our proposed model provides a reduction of $9 \%$ to the best of the randomly generated schedules in the total travel time during the maintenance period of four days. The results show that selecting a correct schedule in case of multiple link closures is crucial in terms of total travel times of network users. It is concluded that randomly generated schedules have a high possibility of leading to longer travel times, whereas schedules obtained using the proposed model is able to reduce travel times. The results also show that the modified ACO can overcome this challenging problem.

For future works, it will be useful to pay attention to not only network users' costs but also the costs arising from the mobilization of maintenance crews and tools. Moreover, the model should also be implemented on larger-scale networks with sets containing higher numbers of roads to be repaired.

\section{CONFLICTS OF INTEREST}

No conflict of interest was declared by the authors. 


\section{REFERENCES}

[1] Fwa, T. F., Cheu, R. L., Muntasir, A., "Scheduling of pavement maintenance to minimize traffic delays", Transportation Research Record, 1650: 28-44, (1998).

[2] Martinelli, D. R., Xu, D., "Delay Estimation and Optimal Length for Four-Lane Divided Freeway Workzones", Journal of Transportation Engineering, 122(2): 114-122, (1996).

[3] Chang, Y., Sawaya, O. B., Chang, Y., "A Tabu Search Based Approach for Work Zone Scheduling”, Computer Science, August, (2000).

[4] Cheu, R. L., Wang, Y., Fwa, T. F., "Genetic Algorithm-Simulation Methodology for Pavement Maintenance Scheduling", Computer-Aided Civil and Infrastructure Engineering, 19: 446-455, (2004).

[5] Lu, G., Xiong, Y., Ding, C., Wang, Y., "An optimal schedule for urban road network repair based on the greedy algorithm", Plos One, 11(10): 1-15, (2016).

[6] Ma, J., Cheng, L., Li, D., "Road Maintenance Optimization Model Based on Dynamic Programming in Urban Traffic Network", Journal of Advanced Transportation, (2018).

[7] Yang, D., Zhao, X., Chen, Y., Zhang, X. and Chen, C., "Study on the day-based work zone scheduling problem in urban road networks based on the day-to-day traffic assignment model", Transportation Research Record, 2672(16): 14-22, (2018).

[8] Rey, D., Bar-gera, H., Dixit, V. V, Waller, S. T., "A Branch-and-Price Algorithm for the Bilevel Network Maintenance Scheduling Problem”, Transportation Science Publication, 53(5): 1213-1499, (2019).

[9] Dorigo, M. and Car, G. Di., "Ant Colony Optimization: A New Meta-Heuristic", Proceedings of the 1999 Congress on Evolutionary Computation, Washington D.C., USA, (1999). 